\title{
Body Weight and Muscle Thickness Changes after Hemodialysis: A Pilot Study with Bayesian Approach
}

\author{
Ana Milena Vieira Peixoto, BSc, PT; Jonas R. Dias da Silva, BSc, PT; David Lomanto Couto, MD; and Rafael Pereira, PhD
}

\begin{abstract}
Maintaining adequate body water balance is one aim of hemodialysis that is obtained by changing the blood volume. However, it is known that volume withdrawal is followed by water redistribution between extra- and intra-cellular spaces. In this context, the skeletal muscle tissue represents almost $50 \%$ of the total body mass, and like other soft tissues, consists of about $70 \%-80 \%$ water. Body weight is the main parameter used to monitor the body water change after a hemodialysis session, but it does not allow inferring about the water redistribution between extra and intracellular spaces. Thus, the present study aimed to assess the immediate impact of hemodialysis on body weight and the thickness of the rectus femoris muscle. Fifteen male patients with end-stage renal disease took part in the study. Muscle thickness, measured with ultrasound imaging, and body weight and were measured before (Pre) and after (Post) (within 5 to 10 minutes after) an hemodialysis session. Paired t-test was used to compare Pre and Post measures, and Bayesian analysis was applied to check the probability to replicate the same results (ie, the magnitude of the evidence). Our results indicated that body weight, but not rectus femoris muscle thickness, is significantly reduced immediately after hemodialysis. The rectus femoris muscle thickness seems not to be a reliable parameter to infer water redistribution after hemodialysis session.
\end{abstract}

Keywords: Ultrasound; Bayesian analysis; Hydroelectrolytic balance

$\mathrm{D}$ ialysis is an indispensable procedure for people with end-stage renal disease and aims to remove metabolites and maintain the hydroelectrolytic balance, at the cost of a large volume of liquid withdrawal from the extracellular space (blood vessels or peritoneum). Reductions of approximately 2000 grams in body weight are common after hemodialysis sessions lasting approximately 4 hours. ${ }^{1}$

It is known that any change in body water volume is associated with a redistribution of body fluids between the intra- and extra-cellular compartments. ${ }^{1,2}$ In this context, the skeletal muscle tissue has a major contribution, since it represents almost $50 \%$ of the total body mass, and like other soft tissues, consists of about $70 \%-80 \%$ water. ${ }^{3}$ Therefore, it is plausible to hypothesize that the dialysis-induced volume withdrawal could reduce muscle thickness acutely, which could serve as a useful indicator of redistribution of water volume in tissues. The present study aimed to assess the immediate impact of hemodialysis on body weight and the thickness of the rectus femoris muscle.

\section{Methods}

Fifteen male patients (9 Black, 1 White, and 5 mulatto) took part in the study. The average age of the study participants was $44.13 \pm 9.32$ years old. The participants were diagnosed with end-stage renal disease and had been undergoing hemodialysis treatment for at least 6 months. The dialysis
Corresponding Author: Dr. Rafael Pereira, PhD, Integrative Physiology Research Center, Department of Biological Sciences, Universidade Estadual do Sudoeste da Bahia (UESB), Jequie, 452 I0-506, Bahia, Brazil, Email: rafaelpereira@uesb.edu.br
Received: January 15, 2021

Revised: May II, 202 I

Accepted: May 25, 2021

doi: $10.3121 / \mathrm{cmr} .2021 .1569$ 
Table 1. Comparisons from body weight and rectus femoris muscle thickness obtained PRE and POST hemodialysis session.

\begin{tabular}{lcccccc}
\hline Variable & Pre & Post & $\begin{array}{c}\text { Mean difference } \\
(95 \% \text { Cl) }\end{array}$ & P value & BF10 & $\begin{array}{c}\text { Probability } \\
(\%)\end{array}$ \\
\hline BW (kg) & $67.2 \pm 10.4$ & $65.1 \pm 10.3$ & $\begin{array}{c}-2.09 \\
(-2.75 \text { to }-1.42)\end{array}$ & $<0.001$ & 2221.2 & 99.9 \\
RF muscle thickness $(\mathrm{cm})$ & $1.21 \pm 0.35$ & $1.14 \pm 0.28$ & $\begin{array}{c}-0.07 \\
(-0.15 \text { to } 0.01)\end{array}$ & 0.087 & 1.07 & 51.7 \\
\hline
\end{tabular}

BF, Bayesian Factor; BW, body weight; RF, rectus femoris

time was $71.47 \pm 62.95$ months, and the study was conducted at the Kidney Disease Center of Jequié (CDRJ) - Bahia, Brazil from December 2019 to March 2020. As we developed this as a pilot study with a small sample, only males were included in the studied sample, since female steroid hormones could influence the body water content, which would increase the data variance, compromising the data interpretation. Participants were invited to take part in the study and informed about the study procedures; those who agreed to participate in the study signed an informed consent document. The study was approved by the Research Ethics Committee of the Universidade Estadual do Sudoeste da Bahia.

Patients were evaluated before (Pre) and after (Post) (within 5 to 10 minutes after) a hemodialysis session. The evaluation consisted of body weight, measured on an electronic floor scale (accuracy $0.1 \mathrm{~kg}$ ), and ultrasound image recordings, described as follows.

Transverse ultrasound images of the right rectus femoris were obtained with a B-mode 2-dimensional ultrasound imaging device (Figlabs FP 102). A linear transducer (7.0 MHz, 75-dB gain; Figlabs, L471) was positioned perpendicular to the longitudinal axis of the rectus femoris at the midpoint between the greater trochanter and the proximal end of the patella., ${ }^{4,5}$ During the image recording, the pressure was kept to a minimum to avoid excess compression and distortion, and a generous amount of contact gel was applied. All measurements were performed by a single trained investigator (JRDS), and a set of three consecutive pictures was taken and further analyzed offline.

Measurements were taken on the patient's right leg with the patient lying in a supine position with both knees extended but relaxed and toes pointing toward the ceiling. The rectus femoris muscle thickness was measured on the distance from the fat-muscle interface to the fascia between rectus femoris and vastus intermedius muscles.

Data were presented as mean \pm standard deviation and mean difference (Post minus Pre) and its 95\% confidence interval (95\% CI). Paired $t$-test was used to compare Pre $v s$ Post body weight and rectus femoris muscle thickness measures, and the significance level was set as $P<0.05$. To check the probability to replicate the same results (ie, the magnitude of the evidence), we applied the Bayes Factor (BF) hypothesis testing analyses. ${ }^{6}$ Individual comparisons were based on the default $t$-test with a Cauchy $(0, r=1 / \sqrt{ } 2)$ prior. The outcomes were classified as anecdotal $(\mathrm{BF} 10=1-3)$, moderate (3-10), strong (10-30), very strong (30-100), and extreme $(>100)$ favoring the alternative hypothesis; or anecdotal $(\mathrm{BF} 10=1$ $0.33)$, moderate $(0.33-0.1)$, strong $(0.1-0.03)$, very strong $(0.03-0.01)$, and extreme $(<0.01)$ favoring the null hypothesis (Lee and Wagenmakers' classification). ${ }^{7,8}$ To calculate the probability of finding the same results again, we divided the actual $\mathrm{BF} 10$ value by $\mathrm{BF} 10+1$. We made all $\mathrm{BF}$ analysis through JAMOVI (Version 1.1.9.0) open stats software (https://www.jamovi.org/).

\section{Results}

Between the studied variables, only body weight reduced significantly after hemodialysis $(P<0.001)$. Rectus femoris muscle thickness reduction was not significant $(P=0.087)$. The BF 10 for body weight indicated high (99.9\%) probability of body weight being affected by hemodialysis, while the $\mathrm{BF}=10$ for rectus femoris muscle thickness indicated anecdotal (51.7\%) probability to be affected by hemodialysis. Table 1 presents the results from frequentist and Bayesian analysis.

\section{Discussion}

Maintaining the adequate body water balance is one aim of hemodialysis that is obtained by changing blood volume (ie, blood volume withdrawal). However, it is known that volume withdrawal is followed by water redistribution between extraand intra-cellular spaces. The blood volume reduction could lead to a hypotensive state, culminating in intradialytic hypotension - the most prevalent - and then, the main intercurrence along hemodialysis.

The use of ultrasound images to monitor volume withdrawal during hemodialysis have been proposed, by measurement of the inferior vena cava diameter (IVCD) with ultrasound, ${ }^{2}$ but it is a complex process that requires a refined technique. Katzarski et $\mathrm{al}^{2}$ identified that body weight, blood pressure, blood volume, and IVCD decreased over the course of hemodialysis, and then increased 2 hours after hemodialysis due to refilling of the intravascular space. Indeed, water from intracellular space is redistributed for intravascular space, 
making it plausible to hypothesize that the measure of muscle thickness could bring insights regarding this event, since muscles consist of about $70 \%-80 \%$ water.

Ultrasound imaging recording and measurement of skeletal muscles thickness requires less refined technique than measurement of the IVCD, and changes in rectus femoris thickness after hemodialysis, if significant and reliable, could be a useful tool to monitor the water balance associated with hemodialysis. However, our results indicated that the rectus femoris thickness was not significantly reduced immediately after hemodialysis, and the Bayesian analysis approach indicated an anecdotal $(51.7 \%)$ probability to be affected by hemodialysis. On the other hand, our results established that body weight is significantly reduced immediately after hemodialysis, confirming a previous report, ${ }^{2}$ also confirming a high $(99.9 \%)$ probability of body weight being affected by hemodialysis.

The hypothesis of rectus femoris thickness measurement as a tool to monitor the water balance associated with a hemodialysis session could be remarkably interesting and useful, but our initial investigation indicated a low probability of this being true. It seems that measurement of IVCD, despite the complexity, could be the most adequate way to monitor volume withdrawal and subsequent intravascular refilling after a hemodialysis session.

\section{Conclusion}

Body weight, but not rectus femoris muscle thickness evaluated by ultrasound imaging, is significantly reduced immediately after hemodialysis. Despite the promising hypothesis, the muscle thickness measure seems not to be a reliable way to monitor the water balance between intra- and extra-cellular spaces, aiming to achieve the intravascular refilling after hemodialysis.

\section{References}

1. Chanchairujira T, Mehta RL. Assessing fluid change in hemodialysis: Whole body versus sum of segmental bioimpedance spectroscopy. Kidney Int. 2001;60(6):2337-2342.

2. Katzarski KS, Nisell J, Randmaa I, Danielsson A, Freyschuss U, Bergström J. A critical evaluation of ultrasound measurement of inferior vena cava diameter in assessing dry weight in normotensive and hypertensive hemodialysis patients. Am J Kidney Dis. 1997;30(4):459-465.

3. Sarvazyan A, Tatarinov A, Sarvazyan N. Ultrasonic assessment of tissue hydration status. Ultrasonics. 2005;43(8):661-671.

4. Thomaes T, Thomis M, Onkelinx S, Coudyzer W, Cornelissen V, Vanhees L. Reliability and validity of the ultrasound technique to measure the rectus femoris muscle diameter in older CAD-patients. BMC Med Imaging. 2012;12(1):7.
5. Perkisas S, Baudry S, Bauer J, et al. Application of ultrasound for muscle assessment in sarcopenia: towards standardized measurements. European Geriatric Medicine. 2018;9(6):739-757.

6. Gönen M, Johnson WO, Lu Y, Westfall PH. The Bayesian two-sample t test. American Statistician. 2005;59(3):252-257.

7. Jeffreys H. Theory of probability. Oxford, UK: Oxford University Press. 1961.

8. Quintana DS, Williams DR. Bayesian alternatives for common null-hypothesis significance tests in psychiatry: a non-technical guide using JASP. BMC Psychiatry. 2018;18(1):178.

\section{Author Affiliations}

Ana Milena Vieira Peixoto, BSc, PT*; Jonas R. Dias da Silva, BSc, PT*; David Lomanto Couto, MD*; and Rafael Pereira, $P h D^{*}$

*Integrative Physiology Research Center, Research Group in Neuromuscular Physiology, Department of Biological Sciences, Universidade Estadual do Sudoeste da Bahia (UESB), Jequie, Bahia, Brazil 